\title{
Distribution Network Risk Assessment Using Multicriteria Fuzzy Influence Diagram
}

\author{
Aleksandar Janjic $(\mathbb{D}$ \\ University of Nis, Faculty of Electronic Engineering, 18000 Nis, Serbia \\ Correspondence should be addressed to Aleksandar Janjic; aleksandar.janjic@elfak.ni.ac.rs
}

Received 20 May 2018; Accepted 1 August 2018; Published 16 September 2018

Academic Editor: Qi Zeng

Copyright (C) 2018 Aleksandar Janjic. This is an open access article distributed under the Creative Commons Attribution License, which permits unrestricted use, distribution, and reproduction in any medium, provided the original work is properly cited.

\begin{abstract}
Risk assessment of distribution assets is one of the most important factors in the process of network development or maintenance planning decision-making. The process of decision-making is faced with uncertainties, involving technical, financial, safety, environmental, and other operational issues that make standard risk assessment techniques insufficient. Probabilistic uncertainties require appropriate mathematical modeling and quantification when predicting future state of the nature or the value of certain parameters. The paper is proposing a new methodology for the multicriteria risk assessment of the distribution network assets, based on influence diagrams and fuzzy probabilities. Influence diagram has been used to determine all relevant factors concerning risks and their interdependencies are depicted. Fuzzy probabilities are represented by triangular fuzzy numbers with constraints on feasibility of elicited probabilities. This methodology enables the decision process in uncertain environment, with the impact evaluation of each particular distribution asset, or the asset component. The methodology is illustrated on the example of a distribution substation circuit breaker maintenance strategy selection.
\end{abstract}

\section{Introduction}

Maintenance planning, development, and reconstruction of distribution networks are playing the crucial role in the asset management of distribution networks [1,2]. One of the main problems of asset management in distribution companies is to find the best maintenance strategy out of following actions: do nothing and repair only after the breakdown, overhaul, or do the complete replacement of asset. Some activities, like minor or major maintenance, can be performed in a regular time interval, or depending on condition of an asset, but the problem is becoming more complex as alternatives must be evaluated on the basis of several criteria [3]. Some of them are easy to measure (costs and profit), while others can be very difficult to evaluate (public opinion, consequences of outages).

Risk and uncertainties are also present in the process of decision-making, whether it is in presupposed data (consumption increase rate, prices, and preferences) or in decision factors of business environment that affect the process of decision-making. One of the latest approaches is the risk management based maintenance, which evaluates the risk of equipment failure and consequences such failure can produce on the system [4-6]. With the quantification of risk, the most efficient strategy and the optimal risk level for distribution networks assets management can be obtained [6]. In all these approaches, risk is defined as a combination of probability indices and the consequences of failure in the network.

Decision about the optimal level of maintenance depends on several criteria of different nature:

(i) technical criteria

(ii) economic criteria

(iii) health and safety criteria

(iv) environmental impact

(v) public opinion and customer satisfaction

(vi) regulatory requirements

The number and structure of these categories is changing, depending on particular conditions (legislative, regulatory requirements, etc.) but these are basic attributes out of which the others can be derived. Furthermore, the asset management problem is facing the probabilistic uncertainty 
and imprecision when modeling problem structural parameters, including the required goals, constraints, and external influences.

Various theories of imprecise probability include the Dempster-Shafer evidence theory $[7,8]$, the coherent lower prevision theory [9], probability bound analysis [10], and the fuzzy probability [11]. Stochastic nature of parameters and subjective probabilities are often described with interval probabilities or fuzzy sets. Interval and fuzzy probabilities are used when it is hard to model uncertainty by point value probabilities: when little or no information to evaluate them is available, or when several information sources (sensors, individual experts in group decision-making) are combined [12]. Fuzzy modeling can be understood as an extension to interval modeling, and fuzzy probabilities can be characterized by a possibility distribution of probability, representing degree of confidence in that probability expressed by an individual $[13,14]$.

Bayesian networks and Influence diagrams are used as a convenient tool for the large class of engineering problems, while the inherent uncertainty has been modeled by the fuzzification of random variables, and/or prior and conditional probabilities. A comprehensive review of development dealing with imprecise probabilities for the solution of various engineering problems is given in [15]. Fuzzy probabilities are treated as an extension of interval probabilities, emphasizing the correspondence between different $\alpha$-levels and probability boxes. Various engineering analyses are then enabled using min-max operator and extension principle as the basis for the processing of fuzzy information.

In Bayesian networks, uncertainty embodies both sources: aleatoric (random events or uncontrollable variation) and epistemic (as the absence of complete knowledge). Furthermore, fuzzy probabilities, grouped in several fuzzy sets, can be denoted with linguistic terms: "extremely low", "very low", "medium", etc. [16-19]. These terms represent the information granules that are in great extent influenced by the psychological profile of the decision-maker.

In the deterministic case, alternatives and consequences are directly related in terms of criteria. In the presence of uncertainties, there may exist many possible outcomes that can be described quantitatively or qualitatively (through verbal descriptions).

Approaches like Bayesian networks, fault, and events trees are often used to understand and model random events and outcomes, but issues like interdependencies of different criteria in the decision-making process require further attention. New form of description, the influence diagram, that is both a formal description of the problem that can be treated by computers and a simple, easily understood representation is presented in this paper. The formal theory of Influence diagram is given in $[20,21]$, with the evaluation, or solving of influence diagram based on Bayesian networks.

This work introduces a new methodology for the risk assessment in distribution network based on the extension of Influence diagrams with the fuzzy probabilities and different consequence evaluation. Risk assessment is performed in two steps. In the first step, influence diagram has been used to determine all relevant factors influencing risks with the depiction of their interdependencies, together with all possible alternative decisions. In the second step, the set of each particular risk values is calculated as the combination of risk factor occurrence and their consequences. Subjective probabilities are represented as information granules described by linguistic terms and modeled as triangular fuzzy numbers.

In the next section of this paper, both steps of a risk assessment methodology using Bayesian networks and Multicriteria Influence diagram are presented. Building of an influence diagram and the way of solving it are presented. Using joint probability rule, the risk of particular event, for different risk categories, is calculated. In Section 3, the notion of fuzzy probability has been explained and in Section 4 the methodology is illustrated on the case study of the choice of circuit breakers maintenance strategy in one transformer substation.

\section{Risk Assessment Using Influence Diagrams}

2.1. Risk Assessment. Risk assessment, as the first step in the risk management process, attempts to identify possible failure events, evaluate their consequences, determine the probability of their future occurrence, and reduce the detrimental consequences. The usual definition of risk associated with an event $E$ is defined as the product of event probability $p(E)$ and its consequence $\operatorname{Cons}(E)[22,23]$ :

$$
\operatorname{Risk}(E)=p(E) \cdot \operatorname{Cons}(E)
$$

More complex relationships between values introducing empirical scaling parameters $x, y$, and $w$ are presented in the following [24]:

$$
\operatorname{Risk}(E)=p(E)^{y} \cdot w \cdot \operatorname{Cons}(E)^{x}
$$

Calculated value of risk became a crucial factor when deciding about the actions to be performed on distribution asset. However, decisions have to be made in a very uncertain environment. In this paper, a new graphical tool based on Bayesian networks-influence diagrams for risk assessment and decision-making under uncertainty-is proposed. The definition of Bayesian networks is given in the sequel, before proceeding to the risk assessment methodology.

2.2. Bayesian Networks. Bayesian network $(\mathrm{BN})$ is a directed acyclic graph represented with pairs $N=\{(V, E), P\}$. Node $V$ represents random variables (events) and links $E$ between nodes represent a causal dependency. A link from variable $\mathrm{X}$ to variable $\mathrm{Y}$ indicates that $\mathrm{X}$ can cause $\mathrm{Y}$, or, in $\mathrm{BN}$ terminology, $\mathrm{X}$ is a parent of $\mathrm{Y}$, and $\mathrm{Y}$ is a child of $\mathrm{X}$. $P$ is a probability distribution over $V$. Discrete random variables $V=\left\{X_{1}, X_{2}, \ldots, X_{n}\right\}$ are assigned to the nodes variables representing a finite set of mutually exclusive states and annotated with a Conditional Probability Table (CPT) that represents the conditional probability of the variable given the values of its parents in the graph.

The simple Bayes net is presented in Figure 1 with two independent variables, $\mathrm{X}_{1}$ and $\mathrm{X}_{2}$, and dependent variable $\mathrm{Y}$ with appropriate CPT representing probabilities for each 
TABLE 1: Consequences grading scale.

\begin{tabular}{ll}
\hline & Safety consequences \\
\hline 1 & Description \\
2 & No harmful consequences \\
& Minor: failure results in minor system damage but does not cause injury to personnel or allow any kind of \\
3 & exposure to operational or service personnel \\
4 & Major: failure results in a low level of exposure to Personnel, or activates facility alarm system \\
5 & Critical: failure results in minor injury to personnel \\
\hline
\end{tabular}

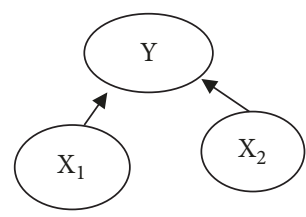

FIgURE 1: Bayes net with two independent variables.

possible state of the nature of variable $\mathrm{Y}$, or event, in the risk assessment terminology.

The solving of $\mathrm{BN}$ is based on four rules, including conditional independence, joint probability, marginalization, and Bayesian rule, presented respectively in the following expressions $[21,22]$.

\section{Conditional Independence}

$$
P\left(X_{1}, X_{2}, \ldots, X_{n}\right)=\prod_{i=1}^{n} P\left(X_{i} / \text { Parents }\left(X_{i}\right)\right)
$$

Joint Probability

$$
P\left(Y=y_{i}, \mathrm{X}=x_{i}\right)=P\left(X=x_{i}\right) \cdot P\left(Y=y_{i} / \mathrm{X}=\mathrm{x}_{i}\right)
$$

Marginalization Rule

$$
P\left(Y=y_{i}\right)=\sum_{i} P\left(X=x_{i}\right) \cdot P\left(Y=y_{i} / \mathrm{X}=\mathrm{x}_{i}\right)
$$

Bayesian Rule

$$
P\left(X=x_{i} / \mathrm{Y}=\mathrm{y}_{j}\right)=\frac{P\left(X=x_{i}\right) \cdot P\left(\mathrm{Y}=\mathrm{y}_{j} / X=x_{i}\right)}{P\left(Y=y_{j}\right)}
$$

Using expressions (3)-(5), the probability of each possible state $j$ out of $n$ possible states of variable $Y$ can be determined. After the calculation of probabilities, the following step in the methodology is to calculate the risk, using expressions (1) and (2), or more generally:

$$
R_{i}=f\left(C\left(Y_{i}\right), P\left(Y_{i}\right)\right)
$$

In order to incorporate the risk, $\mathrm{BN}$ is extended by two more nodes: consequence node $C(Y)$ and risk value node $R$ (Figure 2).

For instance, consequences grades for personal safety can be expressed by numerical grades, described in Table 1 .

Risk value node is represented by the n-dimensional array, with elements calculated from (7).

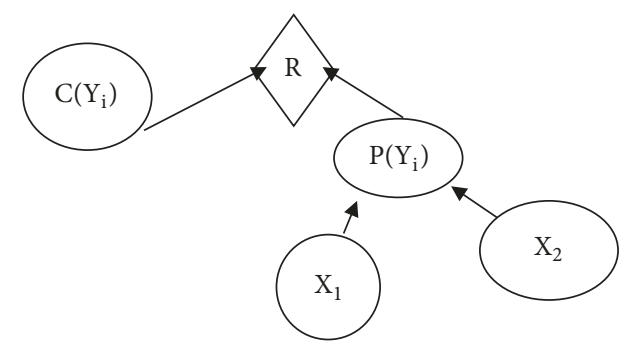

FIGURE 2: Extension of the Bayes net for the risk assessment.

2.3. Influence Diagrams. A generalization of a $\mathrm{BN}$ is the Influence diagram (ID), proposed by Howard and Matheson [21], as a tool to simplify modeling and analysis of decision trees, allowing not only probabilistic inference but also the graphical representation of decision-making problems. Like in $\mathrm{BN}$, the input and output values of a node in an ID are based on the Bayesian theorem, allowing a user to make inferences with limited available information. Besides the chance nodes of BN, ID also contains decision nodes and utility nodes, depicting available information at the time of making a decision, and the degree of influence of each variable on other variables and decisions. Unlike a decision tree that shows more details of possible paths, ID shows dependencies among variables more clearly. IDs are particularly useful in creating computer-based models that describe a system or as descriptions of decision maker's mental models to assess the impact of their actions.

ID tries to capture system representation in a form that can be communicated to others, through several graphical symbols. A circle depicts an exogenous variable (an external influence) whose values are not affected by previous decisions. A rectangle depicts a decision, while intermediate variables depict an endogenous variable whose values are computed as functions of decision and other variables. Chance node (an ellipse) represents a random variable defined by discrete probability distribution. Arrow shows the influence between variables, and dotted arrow shows information being communicated between elements. Finally, value node (a diamond) is a quantitative criterion representing the subject of optimization. The simple example of an ID is presented in Figure 3.

Methods for evaluating and solving IDs are based on Bayes theorem and can be grouped in several categories. They can be (i) converted to decision trees and solved, (ii) solved by 


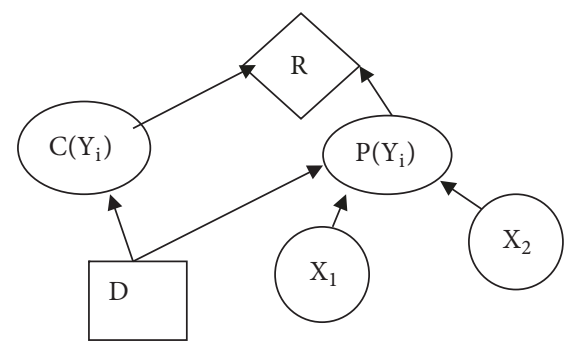

FIGURE 3: Influence diagram for the risk assessment.

the variable elimination algorithms, or (iii) solved by efficient algorithms using graphical structures, like the junction trees [25].

When nodes in the diagram are represented by appropriate fuzzy sets, ID can be solved using fuzzy reasoning [2628]. A commonly used technique for combining fuzzy sets is fuzzy inference system, like the Mamdani type inference. The final step for building an ID model for the risk assessment is to aggregate different risk factors into one diagram. An illustration for the risk assessment ID with two criteria: safety risk (S) and economic risk (E), is presented in Figure 4.

\section{Fuzzy Probability}

Uncertain and subjective probabilities can be granulated in different terms like: improbable or doubtful, but we will draw our attention to special form of probability granulation, focusing on point value with inherent uncertainty: around $30 \%$, around $75 \%$, etc. These probabilities are defined starting from previous works on linguistic probability [29-32] defining similar probability measure for fuzzy probabilities.

Klir [31, 32] introduced a notion of a fuzzy interval defined on $[0,1]$ as a probability granule, that is, a normal fuzzy set on $[0,1]$ with $\alpha$ cuts for all as closed subintervals on $[0,1]$. These fuzzy probabilities will be denoted as $P_{1}, P_{2}, \ldots, P_{n}$ and they may be expressed by the canonical form:

$$
P_{i}(x)= \begin{cases}f_{i}(x), & \mathrm{x} \in[0, \mathrm{~b}) \\ 1, & x \in[b, c] \\ g_{i}(x), & x \in(c, d] \\ 0, & \text { otherwise }\end{cases}
$$

where $a, b, c, d \in[0,1], a \leq b \leq c \leq d$, and $f_{i}$ and $g_{i}$ are strictly increasing right continuous and strictly decreasing left continuous real valued function, respectively. The $\alpha$-cuts of $P_{i}$ are expressed for all $a \in[0,1]$ :

$$
P_{i}^{\alpha}= \begin{cases}{\left[f_{i}^{-1}(\alpha), \mathrm{g}_{i}^{-1}(\alpha)\right],} & \alpha \in(0,1) \\ {[b, \mathrm{c}],} & \alpha=1\end{cases}
$$

In this paper, we will investigate the elicitation of triangular fuzzy set support-left and right bounds of triangular fuzzy numbers. The support of fuzzy set $A$ is the set of all points $x$ in $X$ such that $\mu_{A(x)}>0$.
Consider a discrete random variable $X$ with values in the set $X=\left\{x_{i}, i \in N_{n}\right\}$. We will assume that the probabilities of this random variables $P\left(x_{i}\right)$ are assessed approximately, by a triangular fuzzy number:

$$
\mu_{P_{i}}(\mathrm{x})= \begin{cases}0, & x<a_{i} \\ \frac{x-a_{i}}{b_{i}-c_{i}}, & a_{i}<x<b_{i} \\ \frac{c_{i}-x}{c_{i}-b_{i}}, & b_{i}<x<c_{i} \\ 0, & x>c_{i}\end{cases}
$$

We can interpret these fuzzy numbers as fuzzy probabilities as follows.

Definition 1. Fuzzy numbers $P_{i}=\left[a_{i}, b_{i}, c_{i}\right], i=1, \ldots, n$ are called fuzzy probabilities of $X$ if there are $x_{1} \in$ $\left[a_{1}, c_{1}\right], \ldots, x_{i} \in\left[a_{i}, c_{i}\right], \ldots, x_{n} \in\left[a_{n}, c_{n}\right]$ such that:

$$
\begin{gathered}
\sum_{i=1}^{n} x_{i}=1, \\
\sum_{i=1}^{n} b_{i}=1
\end{gathered}
$$

The set of fuzzy numbers $P$ satisfies (11) if and only if the following conditions hold [33]:

$$
\begin{array}{cr}
c_{i}+a_{1}+\ldots+a_{i-1}+a_{i+1}+\ldots+a_{n} \geq 1, & \forall i \\
a_{i}+c_{1}+\ldots+c_{i-1}+c_{i+1}+\ldots+c_{n} \geq 1, & \forall i
\end{array}
$$

If there are only two fuzzy probabilities $\left[a_{1}, b_{1}, c_{1}\right]$ and $\left[a_{2}, b_{2}, c_{2}\right]$, then $a_{1}+c_{2}=1, a_{2}+c_{1}=1$ and $b_{1}+b_{2}=1$.Let us consider a set of fuzzy numbers $F P=\left\{F P_{i}=\left[a_{i}, b_{i}, c_{i}\right], i=\right.$ $1, \ldots, n\}$. The interval of probability values for every $\alpha$-cut will be denoted as $\left[a_{\alpha, i}, c_{\alpha, i}\right]$. We can interpret these fuzzy numbers as fuzzy probabilities as follows.

Definition 2. Fuzzy numbers $F P_{i}=\left[a_{i}, b_{i}, c_{i}\right]$ are called fuzzy probabilities of $X$ if for $\forall \alpha \in[0,1]$ and $\forall x_{i} \in\left[a_{\alpha, i}, c_{\alpha, i}\right]$ there are $x_{1} \in\left[a_{\alpha, 1}, c_{\alpha, 1}\right], \ldots, x_{i-1} \in\left[a_{\alpha, i-1}, c_{\alpha, i}\right], x_{i+1} \in\left[a_{\alpha, i+1}\right.$, $\left.c_{\alpha, i+1}\right], \ldots, x_{n} \in\left[a_{\alpha, n}, c_{\alpha, n}\right]$ such that:

$$
\sum_{i=1}^{n} x_{i}=1
$$

Lemma 3. The set of fuzzy numbers FP satisfies (13) if and only if the following conditions hold:

$$
\begin{array}{cc}
c_{\alpha, i}+a_{\alpha, 1}+\ldots+a_{\alpha, i-1}+a_{\alpha, i+1}+\ldots+a_{\alpha, n} \leq 1, & \\
a_{\alpha, i}+c_{\alpha, 1}+\ldots+c_{\alpha, i-1}+c_{\alpha, i+1}+\ldots+c_{\alpha, n} \geq 1, &
\end{array} \quad \forall \alpha, \forall i
$$

$\forall \alpha, \forall i$.

Proof.

Sufficient Conditions. If the first part of Lemma 3 holds, then:

$\forall \alpha, \forall i$

$$
\begin{aligned}
x_{i} & +a_{\alpha, 1}+\ldots+a_{\alpha, \mathrm{i}-1}+a_{\alpha, \mathrm{i}+1}+\ldots+a_{\alpha, \mathrm{n}} \leq c_{\alpha, i}+a_{\alpha, 1} \\
& +\ldots+a_{\alpha, \mathrm{i}-1}+a_{\alpha, \mathrm{i}+1}+\ldots+a_{\alpha, \mathrm{n}} \leq 1
\end{aligned}
$$




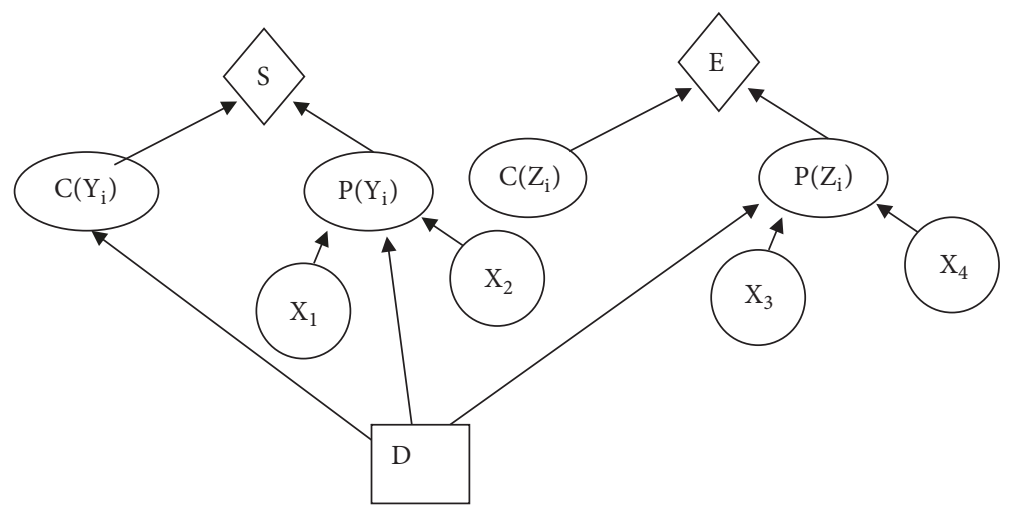

Figure 4: Complete influence diagram with different risk factors.

$\forall \alpha, \forall i$

$$
\begin{aligned}
x_{i} & +c_{\alpha, 1}+\ldots+c_{\alpha, \mathrm{i}-1}+c_{\alpha, \mathrm{i}+1}+\ldots+c_{\alpha, \mathrm{n}} \geq a_{\alpha, i}+c_{\alpha, 1} \\
& +\ldots+c_{\alpha, \mathrm{i}-1}+c_{\alpha, \mathrm{i}+1}+\ldots+c_{\alpha, \mathrm{n}} \geq 1
\end{aligned}
$$

Then, the following expression holds:

$$
\begin{aligned}
x_{i} & +a_{\alpha, 1}+\ldots+a_{\alpha, \mathrm{i}-1}+a_{\alpha, \mathrm{i}+1}+\ldots+a_{\alpha, \mathrm{n}} \leq 1 \\
& \leq x_{i}+c_{\alpha, 1}+\ldots+c_{\alpha, \mathrm{i}-1}+c_{\alpha, \mathrm{i}+1}+\ldots+c_{\alpha, \mathrm{n}}
\end{aligned}
$$

The expression shows that there exist $a_{\alpha, j} \leq x_{j} \leq c_{\alpha, j}, j \in$ $\{1, \ldots n\}, j \neq i$ that satisfies (13).

Necessary Conditions. If the first part of Lemma 3 does not hold, then:

$$
\begin{gathered}
\forall \alpha, \exists i \\
c_{\alpha, i}+a_{\alpha, 1}+\ldots+a_{\alpha, i-1}+a_{\alpha, i+1}+\ldots+a_{\alpha, n}>1 \\
\forall \alpha, \exists i \\
a_{\alpha, i}+c_{\alpha, 1}+\ldots+c_{\alpha, i-1}+c_{\alpha, i+1}+\ldots+c_{\alpha, n}<1 .
\end{gathered}
$$

Then, taking $x_{i}$ as $a_{\alpha, i}$ or $c_{\alpha, i}(13)$ cannot hold.

An alternative definition of fuzzy probabilities can be formulated from two extreme cases of $\alpha=0$ and $\alpha=1$.

Definition 4. Fuzzy numbers $F P_{i}=\left[a_{i}, b_{i}, c_{i}\right]$ are called fuzzy probabilities of $X$ if for and $\forall x_{i} \in\left[a_{i}, c_{i}\right]$ there are $x_{1} \in\left[a_{1}\right.$, $\left.c_{1}\right], \ldots, x_{i-1} \in\left[a_{i-1}, c_{i}\right], x_{i+1} \in\left[a_{i+1}, c_{i+1}\right], \ldots, x_{n} \in\left[a_{n}, c_{n}\right]$ such that:

$$
\begin{gathered}
\sum_{i=1}^{n} x_{i}=1, \\
\sum_{i=1}^{n} b_{i}=1
\end{gathered}
$$

Bayesian networks with fuzzy numbers replacing point value probabilities are proposed [30] defining "Bayesian fuzzy probability" as convex, normal fuzzy set of $[0,1]$. Complementation law has been relaxed in order to extend a partially defined linguistic probability measure, and this method has been successfully used in forensic statistics [29] and risk analysis [18]. More possible scenarios for fuzzifying the Bayesian approach are presented in [15] using nonfuzzy algorithmically efficient reformulation of the Bayesian formula. Although time-consuming, we will implement the corresponding fuzzy version of Bayesian formulas.

The fuzzy counterparts to the standard arithmetic operators are defined using the extension principle. It is possible to derive these operators by examining the effects of interval based calculations at each $\alpha$-cut. The extended operators are defined by (19), using a circled arithmetic operator symbol for the extension of a real arithmetic operator.

Definition 5. For all $a, b \in R^{F}$ a, the extended operators are defined by

$$
\begin{aligned}
& \mu_{A \oplus B}(z)=\sup \min \left(\underset{\substack{\mu_{A}(x), y \\
x+y=z}}{\left.\mu_{B}(y)\right)}\right. \\
& \mu_{A \otimes B}(z)=\sup \min \left(\underset{\substack{A \\
x y=z}}{\left.\mu_{A}(x), \mu_{B}(y)\right)}\right. \\
& \mu_{A-B}(z)=\sup \min \left(\mu_{A}(x), \mu_{B}(y)\right) \\
& \mu_{A \emptyset B}(z)=\sup \min \left(\begin{array}{c}
\left.\mu_{A}(x), \mu_{B}(y)\right) \\
x / y=z
\end{array}\right.
\end{aligned}
$$

From previous definition, two fuzzy Bayes rules analogue to classical crisp number relations are formulated. Operator " $\cong$ " stands for "=" operator.

Fuzzy Joint Probability

$$
\begin{aligned}
P\left(Y=y_{j}, X=x_{i}\right) \cong & P\left(X=x_{i}\right) \\
& \otimes P\left(Y=y_{j} \backslash X=x_{i}\right)
\end{aligned}
$$


TABLE 2: Fuzzy probabilities.

\begin{tabular}{|c|c|c|}
\hline Triangular fuzzy probability number & Description & Notation \\
\hline$\left[\begin{array}{lll}5 & 10 & 15\end{array}\right]$ & Extremely low probability & EL \\
\hline$\left[\begin{array}{lll}15 & 20 & 25\end{array}\right]$ & Low probability & $\mathrm{L}$ \\
\hline$\left[\begin{array}{lll}25 & 30 & 35\end{array}\right]$ & Low to medium & LM \\
\hline [35 40 45] & Medium to low & ML \\
\hline$\left[\begin{array}{lll}45 & 50 & 55\end{array}\right]$ & Medium probability & $\mathrm{M}$ \\
\hline$\left[\begin{array}{lll}5 & 60 & 65\end{array}\right]$ & Medium to high & $\mathrm{MH}$ \\
\hline$\left[\begin{array}{lll}65 & 70 & 75\end{array}\right]$ & High to medium & HM \\
\hline$\left[\begin{array}{lll}75 & 80 & 85\end{array}\right]$ & High probability & $\mathrm{H}$ \\
\hline [85 9005$]$ & Extremely high probability & $\mathrm{EH}$ \\
\hline
\end{tabular}

TABLE 3: Prior probability of weather states.

\begin{tabular}{lcc}
\hline States & Description & Probability \\
\hline Bad & Severe weather conditions & MM \\
Medium & No extreme temperatures below - 20 degree & LM \\
Good & $\begin{array}{c}\text { Good weather conditions, no extreme } \\
\text { temperatures below -10 degree }\end{array}$ & L \\
\hline
\end{tabular}

Fuzzy Bayes Rule

$$
\begin{aligned}
P\left(X=x_{i} \backslash Y=y_{j}\right) \\
\cong \frac{P\left(X=x_{i}\right) \otimes P\left(Y=y_{j} \backslash X=x_{i}\right)}{P\left(Y=y_{j}\right)}
\end{aligned}
$$

Based on the law of total probability another rule for the fuzzy marginalization can be added, represented by (22).

Fuzzy Marginalization Rule

$$
P\left(Y=y_{j}\right) \cong \sum_{i} P\left(X=x_{i}\right) \otimes P\left(Y=y_{j} \backslash X=x_{i}\right)
$$

Finally, risk can be calculated from

$$
R=\sum_{i}^{n} P\left(C_{i}\right) \otimes C_{i}
$$

It is possible to use any other form of additive, multiplicative, or tabular risk aggregation function. The influence diagram with fuzzy probabilities will be illustrated on a simple case study of maintenance strategy selection.

\section{Case Study}

Risk assessment methodology is illustrated on substation with low oil circuit breakers. The decision has to be made about three possible alternatives: do nothing, perform minor interventions, or do the overhaul and major repair of circuit breakers. The alternatives will be assessed by the risk assessment of two criteria: safety and environment. Both criteria will be evaluated by their risk and then aggregated in the one influence diagram value node.

Two failure modes and normal operating condition of a circuit breaker are taken into account: breaker is in operating conditions (OK), failure to close (Close), when breaker does not close the circuit to conduct current in one or more poles, and failure to open (FO), when breaker does not open the circuit to interrupt current. In the case of the bad weather conditions in the following year, the network condition will worsen due to the increased number of failure, the network loading will increase, and the breaker will be exposed to more severe operation conditions. Due to the uncertainty about the weather forecast, and consequently network technical condition, network maximal demand power (loading), and possible failure modes, probabilities elicited by experts are also uncertain. According to the definition of the fuzzy probabilities, possible probability grades are represented in Table 2.

Prior fuzzy probabilities of ambient conditions and global weather forecast for the next year are given in Table 3.

Conditional probability tables for network condition, circuit breaker failure modes, loading levels, and consequences are represented in Tables 4, 5, 6, and 7, respectively.

Safety and environment criteria evaluations are expressed in numerical grades (from 1 to 5 ) and represented in Table 8.

For $\alpha=1$, the influence diagram becomes the deterministic influence diagram with crisp probability values. The solved diagram with calculated values for three different scenarios and two criteria is represented in Figure 5.

Risk is calculated using the following consequences grades: 1 for no consequences, 2 for minor, and 3 for major consequences, and aggregation of two risks is presented in Table 9 .

It is visible that risk calculated values do not show great variance, and that decision about the future maintenance strategy cannot be easily determined. Therefore, the problem is solved again using fuzzy probabilities and expressions (19)-(22). Results are presented on Figures 6, 7, and 8.

Calculated values for risks for both alternatives with crisp values (Figure 5) and fuzzy probability values 
TABLE 4: Conditional probabilities of network conditions.

\begin{tabular}{lcc}
\hline Weather & States & $\begin{array}{c}\text { Good conditions on MV } \\
\text { network, no increase in } \\
\text { failure rate }\end{array}$ \\
\hline B & MH maintenance, increased number of failures & ML \\
M & MM & MM \\
G & ML & MH \\
\hline
\end{tabular}

TABLE 5: Conditional probabilities of failure modes.

\begin{tabular}{lcccc}
\hline Decision & NC & Ok & Close & \\
\hline Minor & & HM & L & EL \\
Minor & Good & H & EL & EL \\
Major & Bad & H & EL & EL \\
Major & Good & EH & EL & EL \\
Do nothing & Bad & MH & L & L \\
Do nothing & Good & MH & L \\
\hline
\end{tabular}

TABLE 6: Conditional probabilities of network loading levels.

\begin{tabular}{lccc}
\hline Weather & Low Loading & Medium Loading & High Loading \\
\hline Bad & EL & LM & MH \\
Medium & LM & MM & L \\
Good & MH & LM & EL \\
\hline
\end{tabular}

TABLE 7: Conditional probabilities of consequences.

\begin{tabular}{|c|c|c|c|c|c|c|c|}
\hline \multirow{2}{*}{$\frac{\text { Loading }}{\text { Low loading }}$} & \multirow{2}{*}{$\frac{\text { Failure mode }}{\text { OK }}$} & \multicolumn{3}{|c|}{ Safety risk } & \multicolumn{3}{|c|}{ Environmental risk } \\
\hline & & $\mathrm{EH}$ & $\mathrm{EL}$ & Impossible & $\mathrm{H}$ & $\mathrm{EL}$ & EL \\
\hline & Failure to close & $\mathrm{H}$ & EL & EL & $\mathrm{H}$ & EL & EL \\
\hline & Failure to open & $\mathrm{HM}$ & $\mathrm{L}$ & EL & $\mathrm{HM}$ & $\mathrm{L}$ & EL \\
\hline \multirow[t]{3}{*}{ Medium loading } & $\mathrm{OK}$ & $\mathrm{H}$ & EL & $\mathrm{EL}$ & $\mathrm{H}$ & $\mathrm{EL}$ & EL \\
\hline & Failure to close & $\mathrm{HM}$ & $\mathrm{L}$ & EL & $\mathrm{MM}$ & LM & $\mathrm{L}$ \\
\hline & Failure to open & $\mathrm{MH}$ & LM & $\mathrm{EL}$ & $\mathrm{MH}$ & LM & EL \\
\hline \multirow[t]{3}{*}{ High loading } & $\mathrm{OK}$ & $\mathrm{HM}$ & $\mathrm{L}$ & EL & $\mathrm{HM}$ & $\mathrm{L}$ & EL \\
\hline & Failure to close & $\mathrm{MH}$ & $\mathrm{L}$ & $\mathrm{L}$ & $\mathrm{MH}$ & $\mathrm{L}$ & $\mathrm{L}$ \\
\hline & Failure to open & MM & LM & $\mathrm{L}$ & MM & LM & $\mathrm{L}$ \\
\hline
\end{tabular}

TABLE 8: Safety and environment criteria grades.

\begin{tabular}{|c|c|c|}
\hline Grade & Safety risk & Environmental risk \\
\hline 1 & No harmful consequences & $\begin{array}{c}\text { No harmful consequences, } \\
\text { Failure does not allow any release } \\
\text { of chemicals into the } \\
\text { environment }\end{array}$ \\
\hline 2 & $\begin{array}{l}\text { Minor: failure results in minor } \\
\text { system damage but does not cause } \\
\text { injury to personnel }\end{array}$ & $\begin{array}{l}\text { Personnel exposure to harmful } \\
\text { chemicals or radiation or fire }\end{array}$ \\
\hline 3 & $\begin{array}{l}\text { Critical: failure results in minor } \\
\text { injury to personnel }\end{array}$ & $\begin{array}{l}\text { A release of chemical to the } \\
\text { environment }\end{array}$ \\
\hline
\end{tabular}




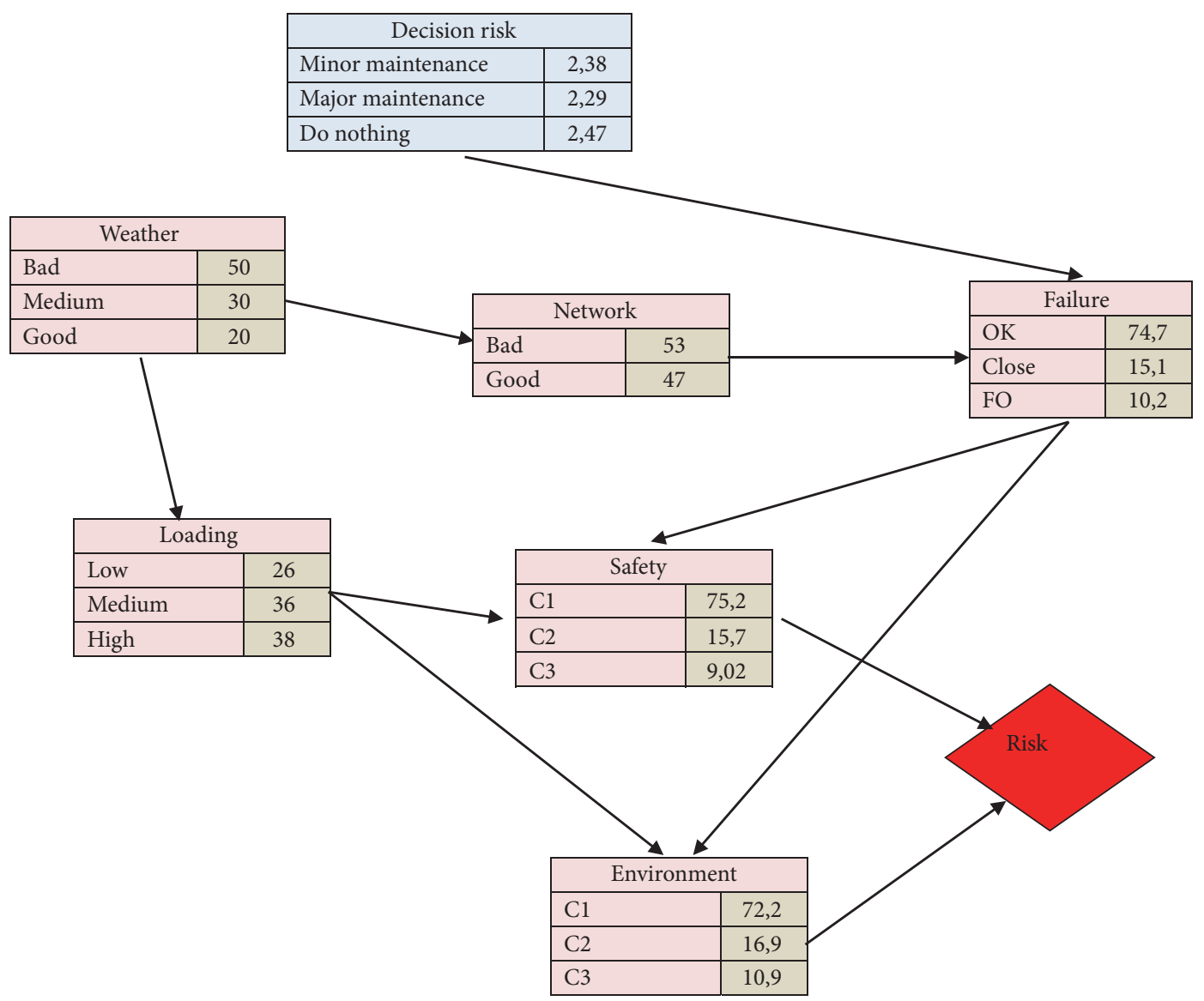

FIGURE 5: Influence diagram with crisp probabilities.
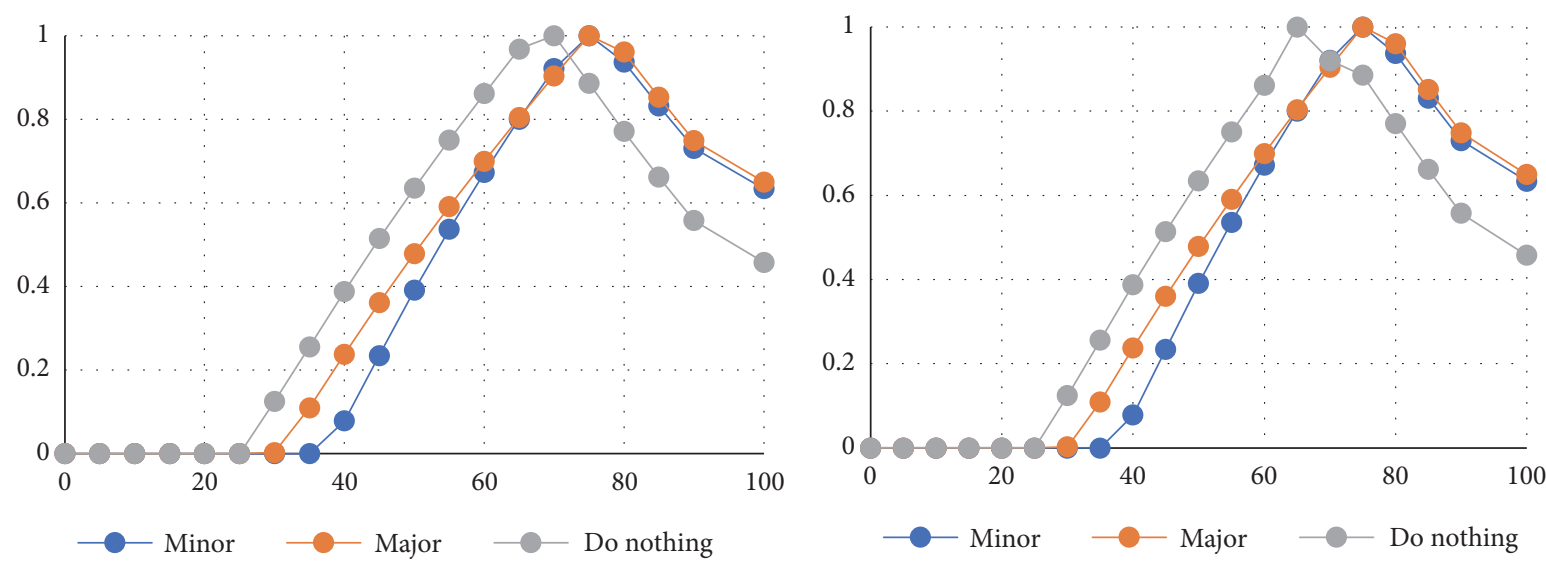

FIGURE 6: Fuzzy probabilities for no consequences state for (a) safety risk and (b) environmental risk.

defuzzified using the centroid method are given in Table 10.

Unlike the crisp calculated values for the risk node, the fuzzy probabilities of possible consequences are highlighting the whole range of future scenarios consequences, facilitating the decision-making process. From Figure 6 it is visible that the "doing nothing" strategy almost doubles the risk for minor and major consequences, while the minor and major maintenance practically do not make any difference.

\section{Conclusions}

Decision-making in maintenance planning is always confronted with several aspects: technical, financial, safety, environmental, and operational ones. Each of these factors can be modeled by the appropriate risk of possible harmful consequences. This paper introduces a new methodology for the optimization of maintenance activities in distribution network, based on the calculation of the risk of the particular 

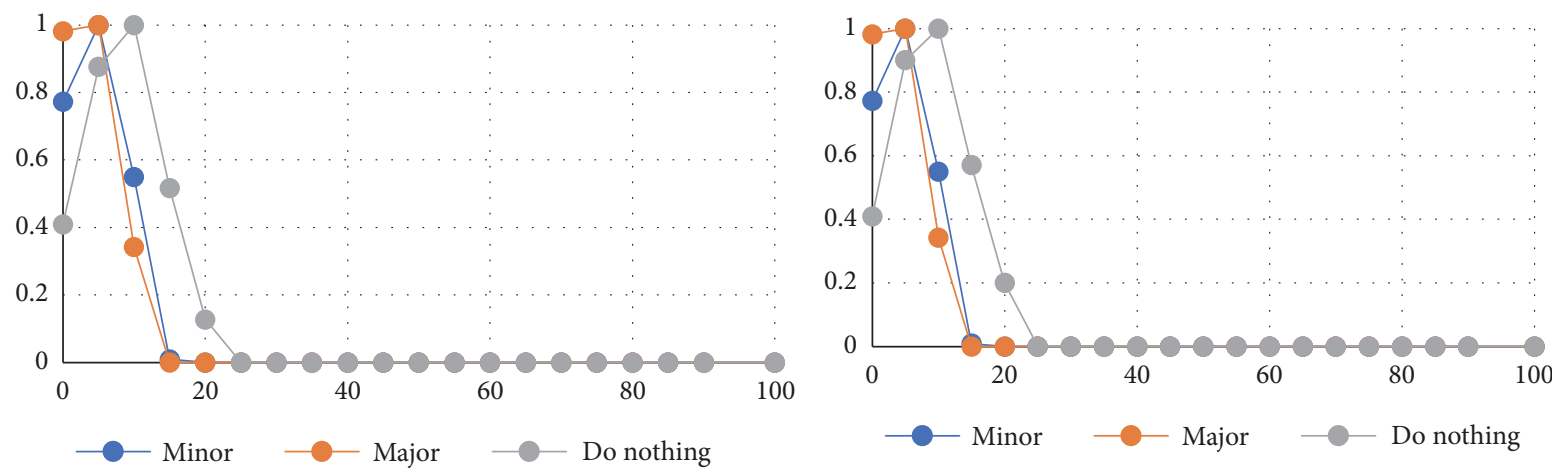

Figure 7: Fuzzy probabilities for minor consequences state for (a) safety risk and (b) environmental risk.
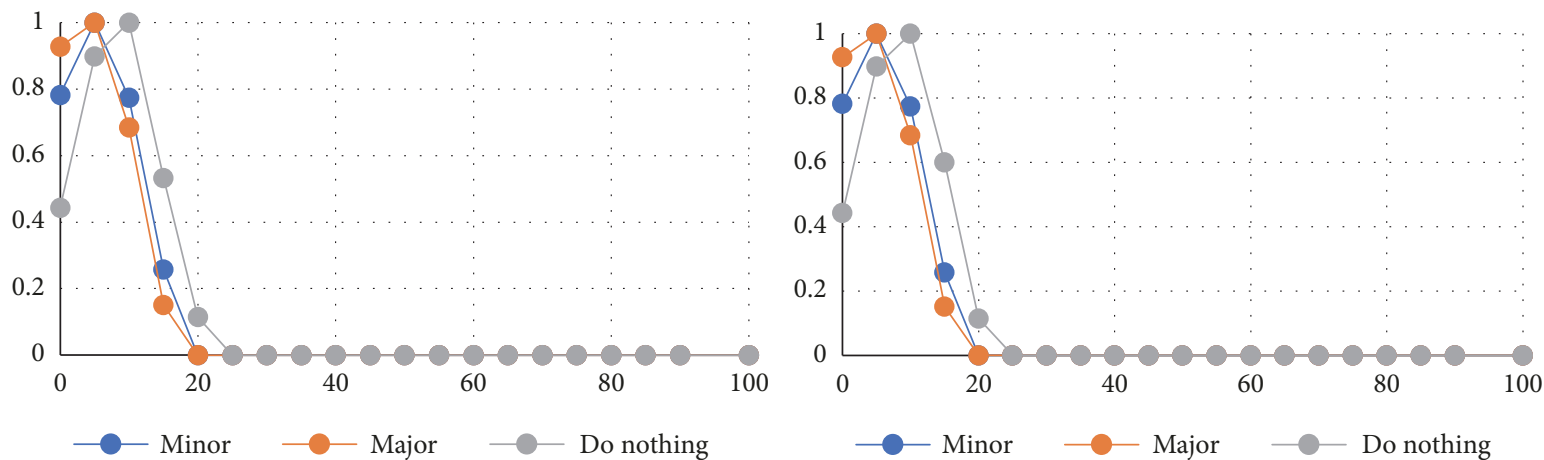

FIGURE 8: Fuzzy probabilities for critical consequences state for (a) safety risk and (b) environmental risk.

TABLE 9: Aggregated risks.

\begin{tabular}{lllllllll}
\hline Safety & 1 & 2 & 3 & 1 & 2 & 3 & 1 & 2 \\
Environment & 1 & 1 & 1 & 2 & 2 & 2 & 3 & 3 \\
Aggregated risk & 1 & 2 & 3 & 4 & 5 & 8 & 7 & 8 \\
\hline
\end{tabular}

TABLE 10: Aggregated risk comparison.

\begin{tabular}{lcc}
\hline & Crisp probability & Fuzzy probability \\
\hline Minor & 2,38 & 2,40 \\
Major & 2,29 & 2,65 \\
Do nothing & 2,48 & 4,12 \\
\hline
\end{tabular}

component, or the overall risk of the distribution object. For this purpose, the Fuzzy Influence diagram has been used, in order to determine all relevant factors influencing risks, and depicting their interdependencies. Probabilistic uncertainties require appropriate mathematical modeling and quantification when predicting future state of the nature or the value of certain parameters and the proposed model enables evaluation of the impact of each particular component of maintenance decision process. Bayesian networks and Influence diagrams are used as a convenient tool for the large class of engineering problems, while the inherent uncertainty has been modeled by the fuzzification of random variables, and/or prior and conditional probabilities. The methodology is illustrated on the example of the choice of circuit breakers maintenance in one distribution transformer substation. This methodology is especially suited for systems with great number of unobservable components, in the presence of uncertainty and lack of operational data, like power distribution system is. The main challenges for the future research are the introduction of fuzzy multicriteria decision analysis for the choice of activity minimizing the overall risk and more flexible representation of fuzzy subjective probabilities, as information granules described by linguistic terms and modeled as triangular fuzzy numbers.

\section{Data Availability}

Data used in the case study are used for simulation purposes. Any calculation result can be obtained from the author by request. 


\section{Conflicts of Interest}

The author declares that he has no conflicts of interest.

\section{References}

[1] G. J. Anders, J. Endrenyi, and C. Yung, "Risk-based planner for asset management," IEEE Computer Applications in Power, vol. 14, no. 4, pp. 718-723, 2001.

[2] S. Natti and M. Kezunovic, "A risk based decision approach for maintenance scheduling strategies for transmission system equipment," in Proceedings of the 10th International Conference on Probabilistic Methods Applied to Power Systems, PMAPS '08, pp. 1-6, 2008.

[3] Y. Jiang, J. D. McCalley, and T. Van Voorhis, "Risk-based resource optimization for transmission system maintenance," IEEE Transactions on Power Systems, vol. 21, no. 3, pp. 1191-1200, 2006.

[4] S. R. Yeddanapudi, Y. Li, J. D. McCalley, A. A. Chowdhury, and W. T. Jewell, "Risk-based allocation of distribution system maintenance resources," IEEE Transactions on Power Systems, vol. 23, no. 2, pp. 287-295, 2008.

[5] A. D. Janjic and D. S. Popovic, "Selective maintenance schedule of distribution networks based on risk management approach," IEEE Transactions on Power Systems, vol. 22, no. 2, pp. 597-604, 2007.

[6] W. Li, Risk Assessment of Power Systems: Models, Methods, and Applications, IEEE Press, 2005.

[7] A. P. Dempster, "Upper and lower probabilities induced by a multivalued mapping," Annals of Mathematical Statistics, vol. 38, pp. 325-339, 1967.

[8] G. Shafer, A Mathematical Theory of Evidence, Princeton University Press, Princeton, NJ, USA, 1976.

[9] P. Walley, Statistical Reasoning with Imprecise Probabilities, Chapman and Hall, London, UK, 1991.

[10] S. Dehaene and J. Mehler, "Cross-linguistic regularities in the frequency of number words," Cognition, vol. 43, no. 1, pp. 1-29, 1992.

[11] B. Möller and M. Beer, Fuzzy Randomness - Uncertainty in Civil Engineering and Computational Mechanics, Springer, Berlin, Germany, 2004.

[12] A. Cano and S. Moral, "Using probability trees to compute marginals with imprecise probabilities," International Journal of Approximate Reasoning, vol. 29, no. 1, pp. 1-46, 2002.

[13] J. J. Buckley, Fuzzy Probabilities. New Approach and Applications, Springer Science \& Business Media, Heidelberg, Germany, 2005.

[14] Y. Pan and G. J. Klir, "Bayesian inference based on intervalvalued prior distributions and likelihoods," Journal of Intelligent \& Fuzzy Systems: Applications in Engineering and Technology, vol. 5, no. 3, pp. 193-203, 1997.

[15] M. Beer, S. Ferson, and V. Kreinovich, "Imprecise probabilities in engineering analyses," Mechanical Systems and Signal Processing, vol. 37, no. 1-2, pp. 4-29, 2013.

[16] C. C. Yang, "Fuzzy Bayesian inference," in Proceedings of the IEEE International Conference on Systems, Man, and Cybernetics, Computational Cybernetics and Simulation, pp. 2707-2712, Orlando, Fla, USA, 1997.

[17] J. Liu, L. M. López, J. Yang, and J. Wang, "Linguistic Assessment Approach for Hierarchical Safety Analysis and Synthesis," in
Intelligent Decision and Policy Making Support Systems, pp. 211230, Springer, 2008.

[18] J. Ren, J. Wang, I. Jenkinson, D. L. Xu, and J. B. Yang, “Bayesian network approach for offshore risk analysis through linguistic variables," China Ocean Engineering, vol. 21, no. 3, pp. 371-388, 2007.

[19] J. Talasova and O. Pavlicek, "Fuzzy Probability Spaces and Their Applications in Decision Making," Austrian Journal of Statistics, vol. 35, no. 2-3, pp. 347-356, 2006.

[20] F. V. Jensen and T. D. Nielsen, Bayesian Networks and Decision Graphs, Springer, 2007.

[21] R. A. Howard and J. E. Matheson, "Influence Diagrams," Decision Analysis, vol. 2, no. 3, pp. 127-143, 2005.

[22] A. Janjić, M. Stanković, and L. Velimirović, "Multi-criteria Influence Diagrams - A Tool for the Sequential Group Risk Assessment," in Granular Computing and Decision-Making, W. Pedrycz and S. M. Chen, Eds., vol. 10 of Studies in Big Data, pp. 165-193, Springer, 2015.

[23] A. Janjic, Z. Stajic, and I. Radovic, "A Practical Inference Engine for Risk Assessment of Power Systems based on Hybrid Fuzzy Influence Diagrams," in Latest Advances in Information Science, Circuits and Systems, 2011.

[24] Z. Zhang, Y. Jiang, and J. McCalley, "Condition based failure rate estimation for power transformers," in Proceedings of the 35th North American Power Symposium, Rolla, Miss, USA, 2003.

[25] F. Jensen, V. Jensen, and S. Dittmer, "From influence diagrams to junction trees," in Uncertainty in Artificial Intelligence (UAI), pp. 367-363, 1994.

[26] N. H. Mateou, A. P. Hadjiprokopis, and A. S. Andreou, "Fuzzy Influence Diagrams: An Alternative Approach to Decision Making Under Uncertainty," in Proceedings of the 2005 International Conference on Computational Intelligence for Modelling, Control and Automation, (CIMCA-IAWTIC '05), 2005.

[27] N. An, J. Liu, and Y. Bai, "Fuzzy influence diagrams: An approach to customer satisfaction measurement," in Proceedings of the 4th International Conference on Fuzzy Systems and Knowledge Discovery, 2007.

[28] L. Hui and X. Y. Ling, "The Traffic Flow Study Based on Fuzzy Influence Diagram Theory," in Proceedings of the 2009 Second International Conference on Intelligent Computation Technology and Automation, 2009.

[29] J. Halliwell, J. Keppens, and Q. Shen, "Linguistic Bayesian Networks for reasoning with subjective probabilities in forensic statistics," in Proceedings of the 5th International Conference of AI and Law, pp. 42-50, Edinburgh, Scotland, 2003.

[30] J. Halliwell and Q. Shen, "Towards a linguistic probability theory," in Proceedings of the 11th International Conference on Fuzzy Sets and Systems (FUZZ-IEEE '02), pp. 596-601, Honolulu, Hawaii, USA, 2002.

[31] G. J. Klir, "Basic Issues of Computing with Granular Probabilities," in Data Mining, Rough Sets and Granular Computing, T. Y. Lin, Y. Y. Yao, and L. A. Zadeh, Eds., Springer Verlag, Germany, 2002.

[32] G. Klir, Uncertainty and Information: Foundations of Generalized Information Theory, John Wiley \& Sons, 2005.

[33] K. Weichselberger and S. Pohlmann, A Methodology for Uncertainty in Knowledge-Based Systems, Springer-Verlag, Germany, 1990. 


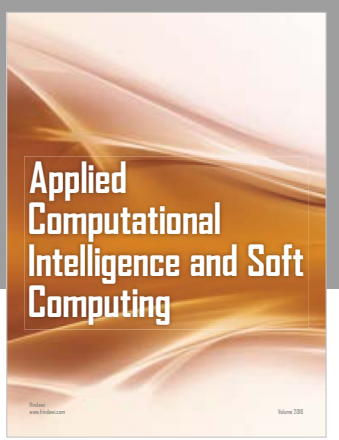

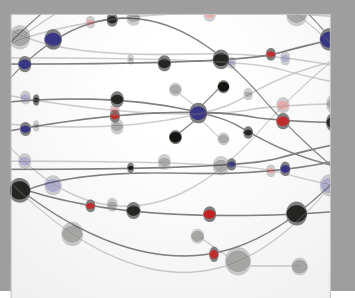

The Scientific World Journal
Submit your manuscripts at

Computing
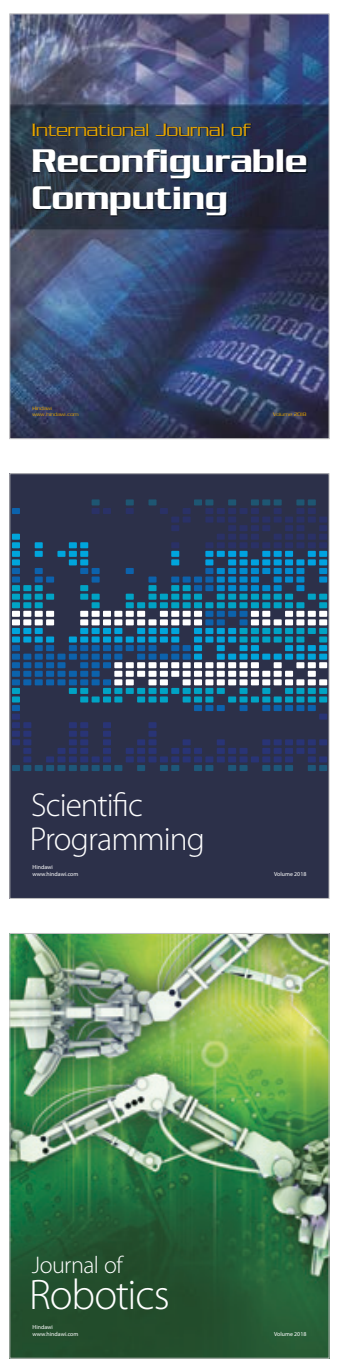

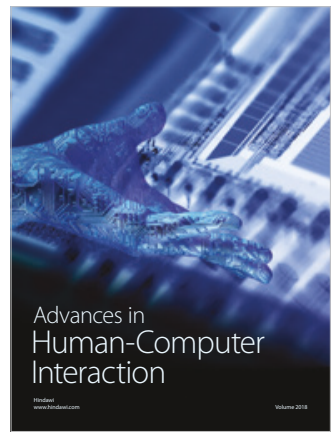

Human-Compute

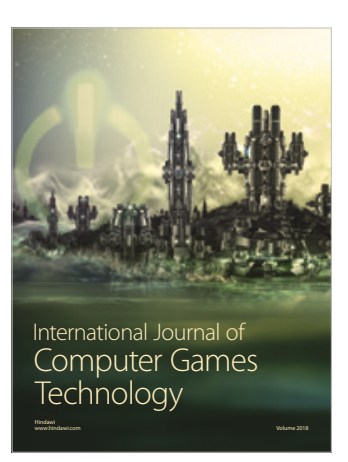

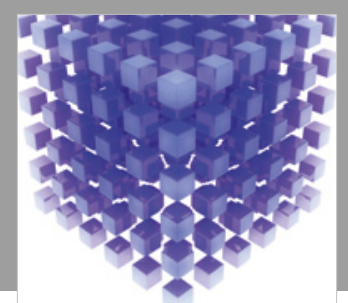

Mathematical Problems in Engineering

\section{Engincering}
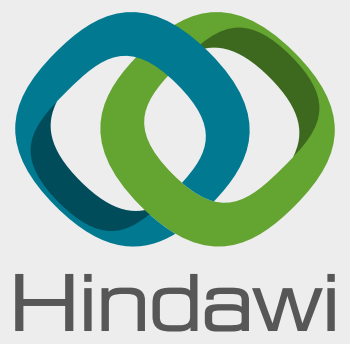

www.hindawi.com
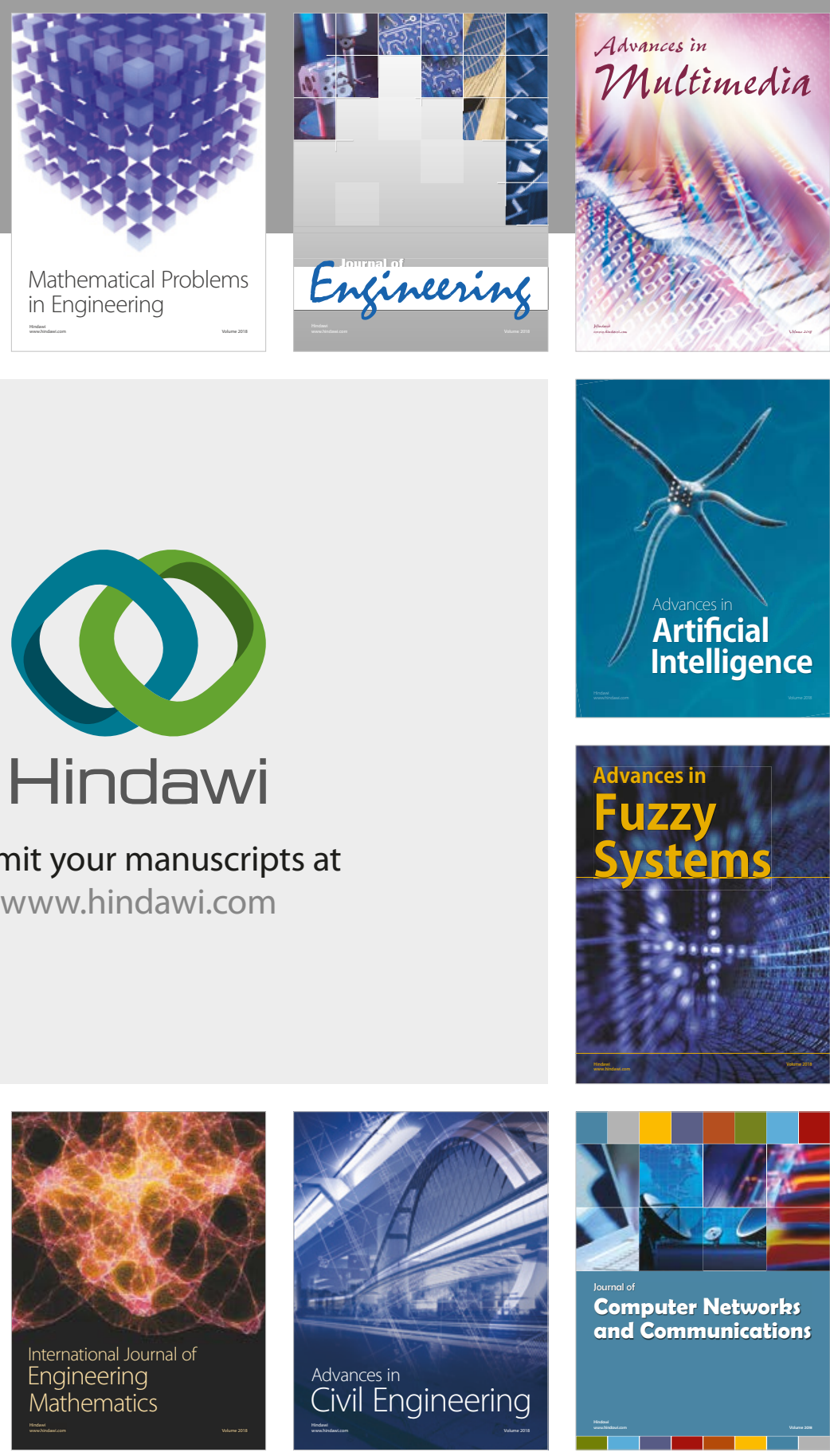

Computer Networks and Communications

Multimedia
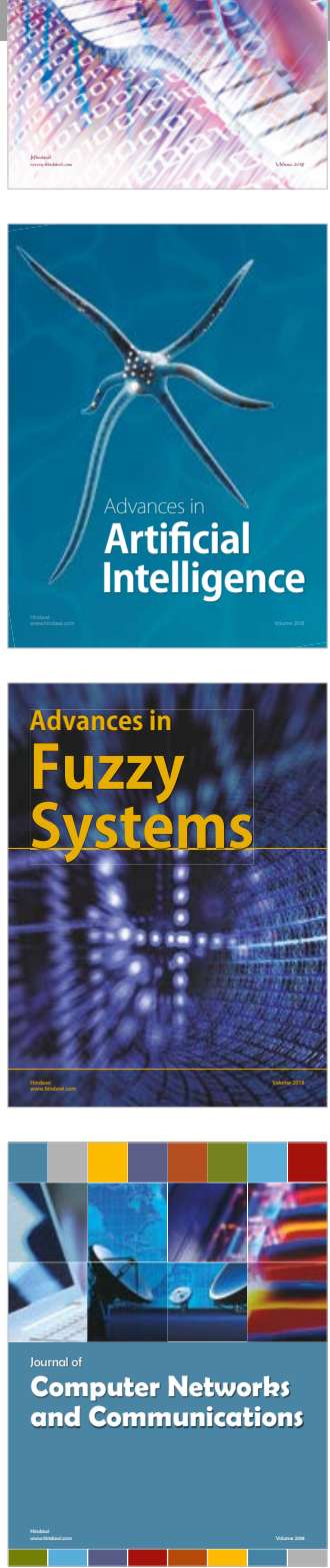

Advances in

Modelling \&

Simulation

in Engineering

interaction

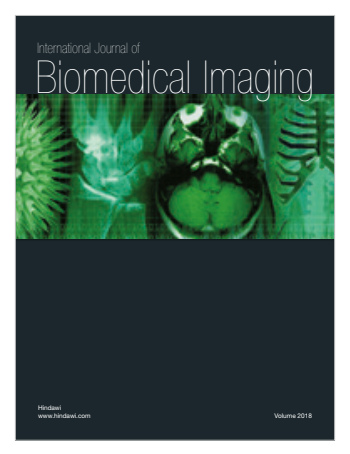

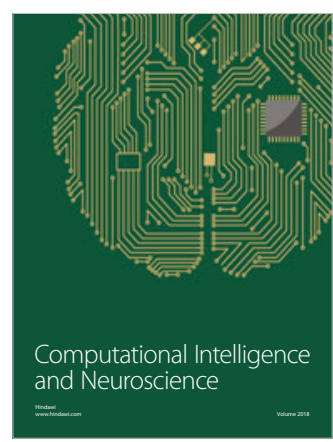

